\title{
Nuclear protein extraction from frozen porcine myocardium
}

\author{
Diederik W. D. Kuster • Daphne Merkus • \\ Huub J. J. Jorna • Dick H. W. Dekkers • \\ Dirk J. Duncker • Adrie J. M. Verhoeven
}

Received: 19 July 2010 /Accepted: 15 October 2010 /Published online: 9 November 2010

(C) University of Navarra 2010

\begin{abstract}
Protocols for the extraction of nuclear proteins have been developed for cultured cells and fresh tissue, but sometimes only frozen tissue is available. We have optimized the homogenization procedure and subsequent fractionation protocol for the preparation of nuclear protein extracts from frozen porcine left ventricular (LV) tissue. This method gave a highly reproducible protein yield $(6.5 \pm 0.7 \%$ of total protein; mean \pm SE, $n=9$ ) and a 6-fold enrichment of the nuclear marker protein B23. The nuclear protein extracts were essentially devoid of cytosolic, myofilament, and histone proteins. Compared to nuclear extracts from fresh LV tissue, some loss of nuclear proteins to the cytosolic fraction was observed. Using this method, we studied the distribution of tyrosinephosphorylated signal transducer and activator of transcription 3 (PY-STAT3) in LV tissue of animals treated with the $\beta$-agonist dobutamine. Upon treat-
\end{abstract}

D. W. D. Kuster · H. J. J. Jorna - D. H. W. Dekkers •

A. J. M. Verhoeven $(\bowtie)$

Department of Biochemistry, Cardiovascular Research Institute COEUR, Erasmus University Medical Center, PO Box 2040, 3000 CA Rotterdam, The Netherlands e-mail: a.verhoeven@erasmusmc.nl

D. W. D. Kuster · D. Merkus - D. J. Duncker Division of Experimental Cardiology, Department of Cardiology, Thoraxcenter, Cardiovascular Research Institute COEUR, Erasmus University Medical Center, Rotterdam, The Netherlands ment, PY-STAT3 increased $30.2 \pm 8.5$-fold in total homogenates, but only $6.9 \pm 2.1$-fold $(n=4, P=0.03$ ) in nuclear protein extracts. Of all PY-STAT3 formed, only a minor fraction appeared in the nuclear fraction. This simple and reproducible protocol yielded nuclear protein extracts that were highly enriched in nuclear proteins with almost complete removal of cytosolic and myofilament proteins. This nuclear protein extraction protocol is therefore well-suited for nuclear proteome analysis of frozen heart tissue collected in biobanks.

Keywords Nuclear extract · Frozen tissue · Transcription factors $\cdot$ Heart tissue
Abbreviations
DPBS
ECL
Dulbecco's phosphate-buffered saline
GAPDH
Enhanced chemiluminescence

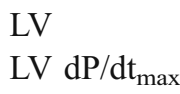
Glyceraldehyde-3-phosphate
dehydrogenase
$\mathrm{LV} \mathrm{dP} / \mathrm{dt}_{\max }$ Maximum rate of rise
of left ventricular pressure
PY-STAT3 Tyrosine-phosphorylated
signal transducer and activator
of transcription 3
SERCA2 Sarcoplasmic/endoplasmic reticulum $\mathrm{Ca}^{2+}$-ATPase 2
SR Sarcoplasmic reticulum
TF Transcription factor 


\section{Introduction}

Preparation of nuclear protein extracts from tissue or cultured cells is an important tool for the study of gene regulation and the transcription factors (TFs) involved. Nuclear protein extracts are widely employed to study DNA binding of TFs in electrophoretic mobility shift assays or to study trafficking of TFs between the cytosol and the nucleus. Nuclear protein extracts are also wellsuited for nuclear proteome analysis as subcellular fractionation greatly reduces the complexity of the protein mixture, thus allowing detection of the low abundant TFs [1, 2, 12]. Preparation of nuclear protein extracts from fresh tissue samples or cultured cells is relatively straightforward. However, it is often desirable to utilize frozen tissue collected over a period of time as for example in biobanks.

In light of these considerations, we set out to develop a method for nuclear protein extraction from frozen cardiac muscle tissue, which is notoriously difficult to homogenize, and we compared this with standardized nuclear protein extraction from fresh heart tissue. Nuclear protein extracts from frozen heart tissue have already been applied for gel shift assays [4, 7, 25, 32] and for the determination of nuclear translocation of TFs $[5,6,10$, 11, 16, 17, 20, 29, 30]. However, homogenization and fractionation protocols used in these studies vary widely. Most of these studies did not report the yield of nuclear proteins or the degree of contamination from other subcellular compartments. Recently, a study was published which analyzed the nuclear extraction from frozen heart tissue with a commercial fractionation kit [22]. The authors found poor purification and high levels of contamination from other subcellular fractions. In the present study, we developed a simple and reproducible nuclear protein extraction method from frozen left ventricular tissue by systematically optimizing the homogenization and fractionation steps. We used cardiac tissue collected over time from pigs, a large laboratory animal, of which the cardiac physiology and anatomy closely resembles that of humans.

\section{Material and methods}

Experimental animals

Studies were performed in accordance with the Guide for the Care and Use of Laboratory Animals (NIH
Publication No. 85-23, revised 1996) and with approval of the Erasmus Medical Center Animal Care Committee. Cardiac tissue was collected from a total of 18 Yorkshire $\times$ Landrace swine. Animals were sedated by an intramuscular injection of a mixture of ketamine and midazolam, anesthetized with intravenous pentobarbital (bolus + continuous infusion), intubated, and ventilated [24, 26]. The thorax and pericardium were opened and the heart was exposed. In a subset of four pigs, dobutamine was infused in consecutive doses of 2.5 and $10 \mu \mathrm{g} / \mathrm{kg} / \mathrm{min}$, for $10 \mathrm{~min}$ each, followed by a 10-min wash out period. Heart rate and LV contractility were determined as previously described $[24,26]$. Then the heart was arrested by application of an electroshock from a 9-V battery [15] and immediately excised. The left ventricle was divided into subendocardial and subepicardial tissue from the anterior, lateral, posterior, and septal area of the left ventricle wall. Subsequently, cardiac tissue was snap-frozen in liquid nitrogen (i.e., within $5 \mathrm{~min}$ of excising the heart) and stored at $-80^{\circ} \mathrm{C}$.

Nuclear protein extraction from frozen tissue

Frozen heart tissue from nine pigs collected over a period of 10 years was used for nuclear protein extraction. Approximately $100 \mathrm{mg}$ of frozen left ventricular subendocardial tissue from the anterior region was pulverized using a Mikro-Dismembrator U (1,700 rpm, 4 min; Sartorius, Nieuwegein, The Netherlands), and the powder was immediately suspended in $4 \mathrm{ml}$ ice-cold Dulbecco's phosphate-buffered saline (DPBS) (Lonza, Verviers, Belgium) containing protease and phosphatase inhibitors (Complete, Roche Diagnostics, Almere, The Netherlands, and phosphatase inhibitor cocktail $1 \&$ 2, Sigma, St. Louis, IL, USA, respectively). All further steps were carried out at $4^{\circ} \mathrm{C}$. After homogenization with a handheld glass-glass Dounce Tissue grinder (5 times loose, 5 times tight pestle; GPE Ltd, Leighton Buzzard, UK), the suspension was centrifuged at $1,850 \times g$ for $15 \mathrm{~min}$. The supernatant was taken as the cytosolic fraction. The pellet was resuspended in 10 volumes of hypotonic CER I buffer (NE-PER kit, Thermo Scientific, Rockford, IL, USA) containing protease (Protease Inhibitor Mix, Amersham Biosciences, Piscataway, NJ, USA) and phosphatase inhibitors and incubated for $10 \mathrm{~min}$. Then, $55 \mu \mathrm{l}$ of detergent-containing CER II solution was added per $100 \mu \mathrm{l}$ of the original pellet volume. This mixture was 
incubated for $1 \mathrm{~min}$, followed by centrifugation at $14,000 \times g$ for $5 \mathrm{~min}$, and the supernatant representing the organelle fraction was collected. The pellet was resuspended in 5 volumes of high-salt NER buffer containing protease and phosphatase inhibitors, and then incubated for $40 \mathrm{~min}$ and vortexed every $10 \mathrm{~min}$. Thereafter, the suspension was centrifuged at $14,000 \times g$ for $10 \mathrm{~min}$, and the supernatant was saved as the nuclear protein extract. The final pellet containing myofilament and histone proteins was resuspended in $500 \mu \mathrm{l}$ DPBS with protease and phosphatase inhibitors. All fractions were snap-frozen in liquid nitrogen and stored at $-80^{\circ} \mathrm{C}$ until further analysis.

\section{Nuclear protein extraction from fresh LV tissue}

Fresh heart tissue from five pigs was used. Nuclear protein extraction protocol was performed with the NE-PER kit (Thermo Scientific) according to the manufacturer's instructions. In short, the heart was excised and a piece of left ventricular subendocardial tissue from the anterior region was taken and rinsed with ice-cold PBS to remove blood. The tissue was cut into small pieces with a razor blade and placed in $1 \mathrm{ml}$ hypotonic CER I buffer containing protease and phosphatase inhibitors. All further steps were carried out at $4{ }^{\circ} \mathrm{C}$. The tissue was homogenized by Teflon-glass Potter homogenization. The suspension was incubated on ice for $10 \mathrm{~min}$. Then, $55 \mu \mathrm{l}$ of detergent-containing CER II solution was added per $1 \mathrm{ml}$ of CER I buffer. This mixture was incubated for $1 \mathrm{~min}$, followed by centrifugation at $14,000 \times \mathrm{g}$ for $5 \mathrm{~min}$, and the supernatant representing the cytosolic fraction was collected. The pellet was resuspended in $500 \mu$ l NER buffer per $1 \mathrm{ml}$ CER I, containing protease and phosphatase inhibitors, and then incubated for $40 \mathrm{~min}$ and vortexed every $10 \mathrm{~min}$. Thereafter, the suspension was centrifuged at $14,000 \times g$ for $10 \mathrm{~min}$, and the supernatant was saved as the nuclear protein extract. The final pellet containing the myofilament and histone protein was resuspended in $500 \mu \mathrm{l}$ DPBS with protease and phosphatase inhibitors. All fractions were snap-frozen in liquid nitrogen and stored at $-80^{\circ} \mathrm{C}$ until further analysis.

\section{Immunohistochemistry}

Cryocoupes $(5 \mu \mathrm{m})$ were cut from left ventricular tissue samples embedded in TissueTek. Sections were probed with B23 antibody (Santa Cruz Biotechnology Inc., Santa Cruz, CA, USA) at 1:4,000 dilution, followed by an anti-mouse IgG horseradish peroxidase (HRP)-conjugated secondary antibody (DAKO, Carpinteria, CA, USA) at 1:100 dilution. Peroxidaselabeled immune complexes were visualized by incubation with 3,3'-diaminobenzidine tetrahydrochloride (DAKO) and the sections were counterstained with hematoxylin.

\section{Immunoblotting}

The protein fractions were prepared in Laemmli's loading buffer [14], and protein concentration was determined using the Bio-Rad RC DC protein assay kit (Bio-Rad, Hercules, CA, USA) using bovine serum albumin as standard. Equal quantities of protein were loaded onto $12 \%$ sodium dodecyl sulfate (SDS)-polyacrylamide gels. After electrophoresis, proteins were transferred overnight to Immun-Blot (for ECL, Bio-Rad) or Immobilon-FL (for Odyssey, Millipore, Bedford, MA, USA) polyvinylidene difluoride membranes. Protein transfer onto the blot was checked with Ponceau S staining.

For enhanced chemiluminescence (ECL), blots were blocked for 30 min with $0.5 \%$ milk powder (Campina Melkunie BV, Eindhoven, The Netherlands) in Trisbuffered saline (TBS) $/ 0.1 \%$ Tween- 20 . Then, primary antibodies were added to the block buffer at the dilutions described in Table 1, and the blots were incubated for $90 \mathrm{~min}$ at room temperature or overnight at $4^{\circ} \mathrm{C}$ in the case of tyrosine-phosphorylated signal transducer and activator of transcription 3 (PY-STAT3). After washing extensively with TBS $/ 0.1 \%$ Tween-20, the blots were incubated with the indicated amount (Table 1) of HRPconjugated secondary antibodies in block buffer for $1 \mathrm{~h}$ or for $3 \mathrm{~h}$ in the case of PY-STAT3. After washing the blots, ECL signals were generated with the Supersignal West Femto Maximum Sensitivity Kit (Thermo Scientific) on Hyperfilm ECL (Amersham).

For quantification, the Odyssey system using fluorescently labeled secondary antibodies was applied as this technique is linear over a larger dynamic range than ECL [19]. Blots were blocked for $1 \mathrm{~h}$ with Odyssey blocking buffer (Li-Cor, Bad Homburg, Germany) diluted 1:1 with DPBS. Blots were incubated for $1 \mathrm{~h}$ with the primary antibodies (Table 1 ) in block buffer containing $0.1 \%$ Tween-20. After extensive washing in DPBS/0.1\% Tween-20, the blots were 
Table 1 Antibodies and dilutions used for immunoblotting and visualization by ECL and Odyssey

\begin{tabular}{|c|c|c|c|c|c|c|}
\hline \multicolumn{3}{|l|}{ Antibody } & \multicolumn{2}{|l|}{ ECL } & \multicolumn{2}{|l|}{ Odyssey } \\
\hline Antigen & Species $^{\mathrm{a}}$ & Supplier & Primary & Secondary ${ }^{\mathrm{b}}$ & Primary & Secondary ${ }^{\mathrm{b}}$ \\
\hline GAPDH & M & Chemicon Int & $1: 3,200,000$ & $1: 8,000$ & $1: 200,000$ & $1: 2,000$ \\
\hline SERCA2 & $\mathrm{R}$ & Santa Cruz & $1: 20,000$ & $1: 8,000$ & $1: 2,000$ & $1: 2,000$ \\
\hline Prohibitin & $\mathrm{R}$ & Abcam & $1: 8,000$ & $1: 8,000$ & $1: 2,000$ & $1: 2,000$ \\
\hline$\alpha$-Actin & M & Sigma & $1: 2,000$ & $1: 2,000$ & $1: 1,000$ & $1: 2,000$ \\
\hline Histone H3 & $\mathrm{R}$ & Eurogentec & $1: 200,000$ & $1: 8,000$ & $1: 100,000$ & $1: 2,000$ \\
\hline B23 & M & Santa Cruz & $1: 100,000$ & $1: 8,000$ & $1: 8,000$ & $1: 2,000$ \\
\hline PY-STAT3 & M & Cell Signaling & $1: 500$ & $1: 2,000$ & - & - \\
\hline
\end{tabular}

\footnotetext{
${ }^{\mathrm{a}}$ Mouse (M), rabbit (R) antibodies
}

${ }^{\mathrm{b}}$ Secondary antibodies for ECL were HRP-conjugated goat anti-mouse or anti-rabbit IgG from Thermo Scientific, and for Odyssey, the secondary antibodies were IRDye800CW-conjugated goat anti-mouse or anti-rabbit IgG from Li-Cor

incubated with IRDye800CW-coupled secondary antibodies (Table 1) for $1 \mathrm{~h}$ in block buffer containing $0.1 \%$ Tween- 20 and $0.01 \%$ SDS. Finally, images were generated by scanning the blots on the Odyssey Infrared Imaging System (Li-Cor).

Statistical analysis

Results are expressed as mean \pm SE. Unpaired Student's $t$ test was used to compare means. Results were considered statistically significant at $P<0.05$ (two-tailed).

\section{Results}

Nuclear protein extraction procedure

To follow the nuclear proteins during homogenization and fractionation and to determine the recovery of nuclear proteins in the nuclear protein extract, we used the abundant nuclear protein nucleophosmin (B23) as a nuclear marker protein [9, 27]. By immunohistochemistry on frozen cardiac tissue, we showed that localization of B23 is almost completely restricted to the nuclei (Fig. 1).

Homogenization of the frozen LV tissue was found to be a critical step in the nuclear protein extraction protocol. Following Polytron homogenization as described by Lin et al. [17] and Gurusamy et al. [11], most of the B23 protein appeared in the cytosolic fraction (data not shown). This was prevented by more gentle homogenization of heart tissue cut into small pieces, using a handheld glass-glass Dounce homogenizer. However, this method resulted in a large intra-assay variation in the amount of total nuclear protein $(7.1 \pm 1.6 \%$, range $2.6-14.6 \% ; n=8)$ and the amount of $\mathrm{B} 23$ recovered in the nuclear fraction as determined by immunoblotting (6.6 \pm 1.9 arbitrary units, range $2.9-19.3 ; n=8$ ). The reproducibility of total protein and B23 recovery in the nuclear protein fraction was markedly improved upon introduction of a tissue pulverization step at liquid nitrogen temperature before Dounce homogenization in PBS (see below).

Next, we tested several fractionation protocols based on differential centrifugation at different sucrose densities $[8,13]$. In our hands, these laborious protocols gave variable degrees of B23 and total protein recovery in the nuclear fraction (data not shown). Instead, we tested whether the commercial nuclear protein extraction kit from Thermo Scientific, which was specifically designed to isolate nuclear and cytoplasmic proteins from fresh tissue, could be made suitable for frozen tissue. After pulverization and homogenization in PBS, we introduced an additional centrifugation step, which leaves the contents of the nuclei damaged by freeze-thawing in the soluble fraction together with the cytosolic proteins (further denoted as the cytosolic fraction). The pellet containing the nuclei was then resuspended in a small volume of CER I buffer, and the extraction procedure was continued according to the manufacturer's instructions. The fractionation protocol is based on swelling of the cells and organelles in hypotonic buffer, followed by 


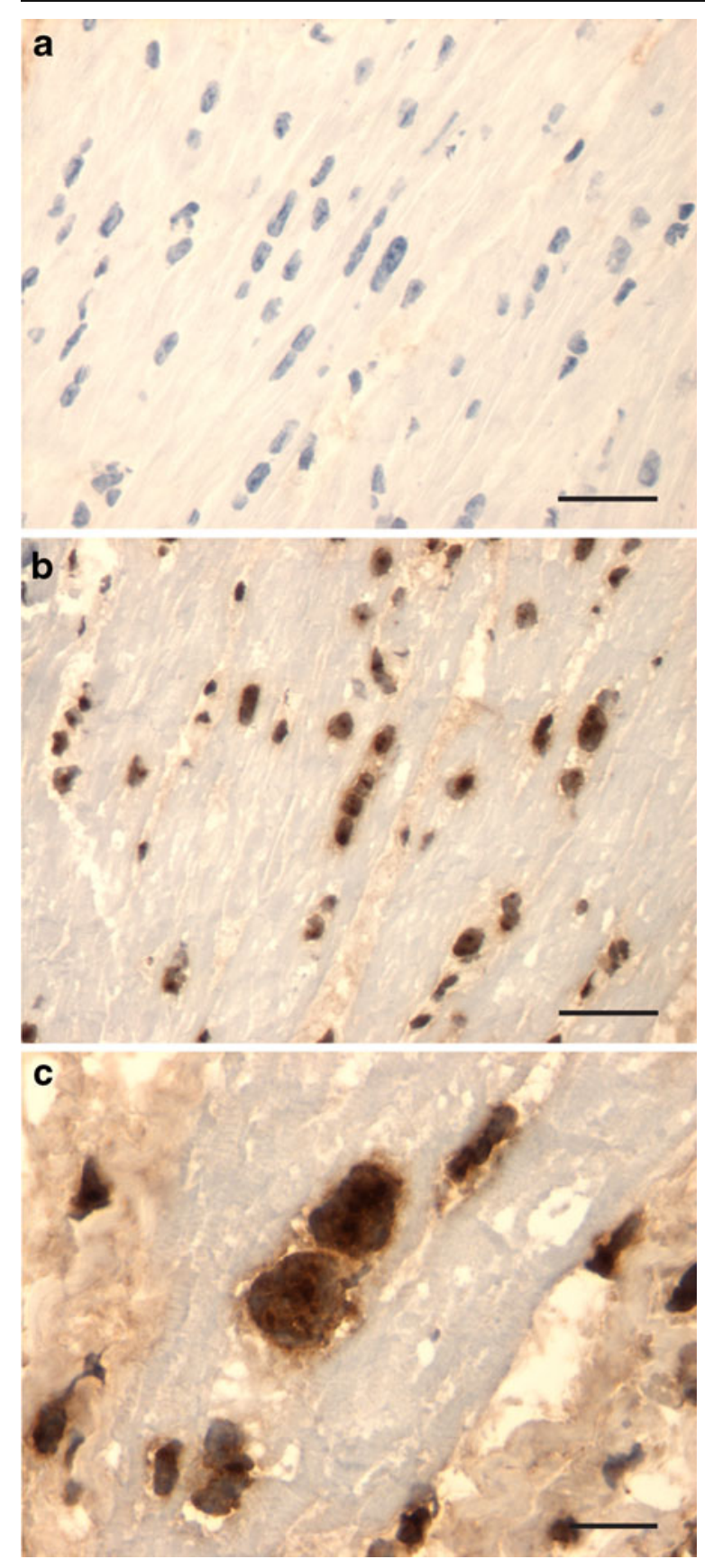

Fig. 1 Subcellular localization of B23 in cardiac tissue. Cryocoupes of cardiac tissue were immunostained (brown) without (a) or with anti-B23 antibody (b, c) and counterstained with hematoxylin (blue). Bar represents $50 \mu \mathrm{m}(\mathbf{a}, \mathbf{b})$ or $10 \mu \mathrm{m}(\mathbf{c})$

bursting of the swollen cells and organelles by detergent. The nucleus is spared from this hypotonic swelling as its nuclear pores allow for the free diffusion of water and electrolytes in and out of the nucleus. The nuclear proteins were extracted from the nucleus by incubation in high-salt buffer. This homogenization and fractionation protocol is relatively simple and showed the highest reproducibility of all the methods tested. A schematic overview of the overall nuclear protein extraction procedure on frozen LV tissue is depicted in Fig. 2a and compared with a standard nuclear protein extraction protocol on fresh cardiac tissue (Fig. 2b). The final pellet fraction left after incubation with nuclear extraction reagent principally contains myofilament proteins and also histone proteins as the salt concentration is too low to enable their extraction from the DNA [28].

Nuclear protein enrichment and contamination

Nuclear fractions were obtained that contained $6.5 \pm 0.7 \%$ (range 3.9-8.6\%, $n=9$ ) of the total protein (Fig. 2a) with more than 6-fold enrichment of B23 (Table 2). B23 enrichment was similar in nuclear protein extracts from frozen and fresh tissues (Table 2). However, compared to the extraction procedure on fresh cardiac tissue, the total protein yield in the nuclear fraction was significantly lower $(P<0.001$, Fig. 2$)$. In extracts from frozen tissue, more B23 was present in the cytosolic fraction than with fresh tissue, probably due to damage of some nuclei during freezing and thawing (Fig. 3). As expected from the relative content of cytosolic and myofilamental proteins in heart tissue, most of the total cellular protein was recovered in the cytosolic and final pellet fractions independent of whether frozen or fresh tissue was used. The relative recovery in these fractions differed between frozen and fresh tissues. It should be noted, however, that due to freeze-thawing, the cytosolic fraction is likely to be contaminated with some proteins derived not only from nuclei but also from damaged organelles. With fresh tissue and using the NE-PER kit, organelle marker proteins ended up in the cytosolic fraction as expected. Total protein recovery in all fractions combined was similar for frozen and fresh tissue extracts $(78.5 \pm 2.7 \%$ vs. $80.6 \pm 6.3 \%)$.

To determine the contamination of the nuclear protein extracts from frozen tissue with proteins from other subcellular compartments, we tested each fraction for the presence of different subcellular marker proteins (Fig. 4). Glyceraldehyde-3-phosphate dehydrogenase (GAPDH) ended up almost exclusively in the cytosolic fraction, with no detectable contamination of the nuclear protein extract. The SR and mitochondria 


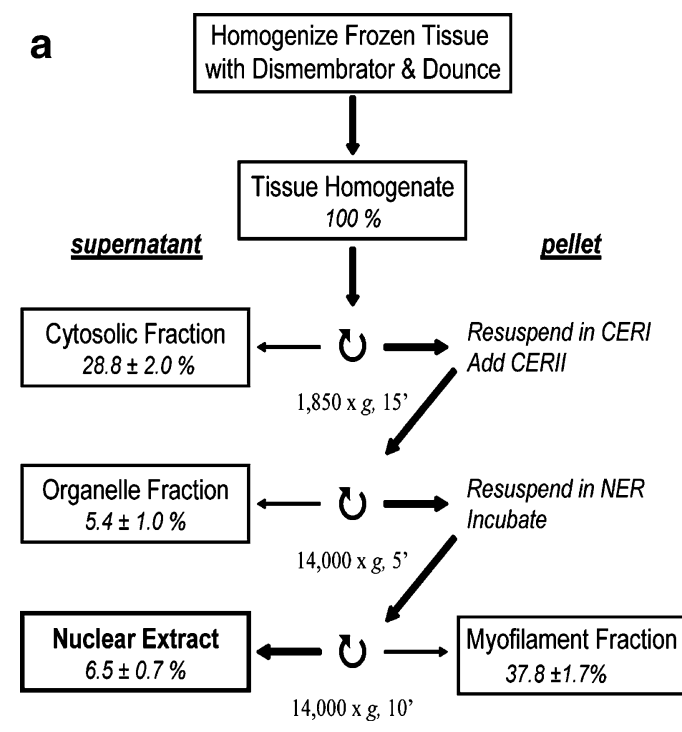

Fig. 2 Nuclear protein extraction protocols from frozen and fresh cardiac tissues. Protocols for the extraction of nuclear protein from frozen (a) and fresh (b) LV tissues are schematically depicted. The amount of protein in each fraction is expressed as a

markers SERCA2 and prohibitin were 8- and 6-fold enriched in the organelle fraction. Nevertheless, some SERCA2 and prohibitin were detected in the nuclear fraction, but these represented only a small percentage (Table 2). Similar results were obtained using ATP

Table 2 Quantification of marker proteins in their specific marker compartment and in the nuclear protein extract

\begin{tabular}{llll}
\hline & \multicolumn{2}{l}{ Marker compartment } & \multirow{2}{*}{ Nuclear extract } \\
\cline { 2 - 3 } & Compartment & RE & RE \\
\hline Frozen tissue & & & \\
GAPDH & Cytosol & $2.5 \pm 0.3$ & $0.0 \pm 0.0$ \\
SERCA2 & Organelles & $8.0 \pm 1.6$ & $0.9 \pm 0.3$ \\
Prohibitin & Organelles & $5.8 \pm 0.8$ & $0.7 \pm 0.2$ \\
$\alpha$-Actin & Final pellet & $3.0 \pm 0.1$ & $0.1 \pm 0.1$ \\
Histone H3 & Final pellet & $4.7 \pm 0.4$ & $0.2 \pm 0.1$ \\
B23 & Nuclei & $6.2 \pm 1.7$ & $6.2 \pm 1.7$ \\
Fresh tissue & & & \\
B23 & Nuclei & $7.6 \pm 1.6$ & $7.6 \pm 1.6$ \\
\hline
\end{tabular}

Data are mean \pm SE from four independent experiments. Quantitative data were obtained with the Odyssey system

$R E$ relative enrichment - the fold increase in protein abundance relative to the total homogenate

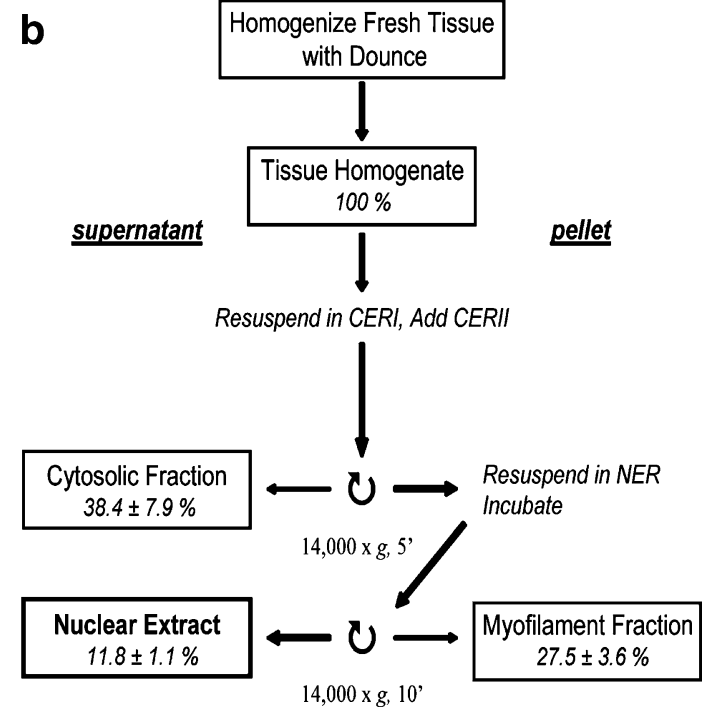

percentage of total protein in the homogenate. Data are mean $\pm \mathrm{SE}$ for nine (frozen) and five (fresh) extractions performed on independent LV tissue samples

synthase subunit $\beta$ as marker for mitochondrial marker (data not shown). The myofilament marker $\alpha$-actin was recovered almost exclusively in the final pellet fraction, with only a minor contamination in the nuclear protein extract. The nucleosome marker histone $\mathrm{H} 3$ fractionated almost exclusively to the final pellet fraction, which is expected as higher salt concentrations are needed to extract histones from the DNA [28].

\section{Nuclear appearance of PY-STAT3}

We employed our method to address the question whether phosphorylation of the TF STAT3 to PYSTAT3 leads to its nuclear accumulation, where it may affect gene expression. In recent years, evidence has emerged that phosphorylation of STAT3 does not necessarily lead to nuclear translocation [21, 23]. It is therefore necessary to demonstrate the nuclear accumulation of PY-STAT3 when studying PY-STAT3dependent gene expression. Stimulation of heart tissue with $\beta$-adrenergic agonists leads to STAT3 phosphorylation $[18,31]$ and increased transcriptional activity [31]. Intravenous infusion with the $\beta$ adrenergic agonist dobutamine in swine resulted in a significant increase in heart rate (from $121 \pm 10$ before 


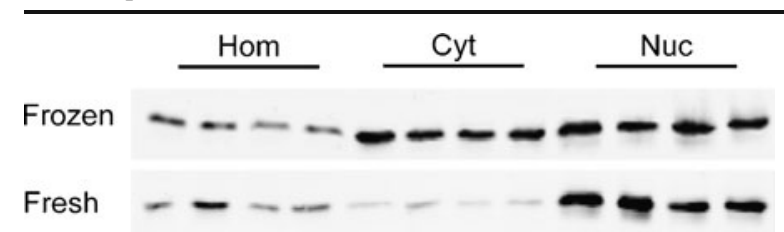

Fig. 3 Distribution of B23 in the cytosolic and nuclear fractions from fresh and frozen tissues. Fractionation was performed on four different frozen and fresh LV tissue samples. Of each fraction, $10 \mu \mathrm{g}$ was analyzed by immunoblotting. Hom total homogenate, Cyt cytosolic fraction, Nuc nuclear protein extract

to $170 \pm 7 \mathrm{bpm}$ during dobutamine infusion, $n=4)$ and $\mathrm{LV}$ contractility $\left(\mathrm{LV} \mathrm{dP} / \mathrm{dt}_{\max }\right.$ from $1,778 \pm 223$ before to $4,955 \pm 1,052 \mathrm{mmHg} / \mathrm{s}$ during dobutamine infusion, $n=4)$. From the treated and control sham-operated animals, total homogenates and nuclear protein extracts were prepared from frozen LV tissue samples. Tyrosine-phosphorylated STAT3 was 30.2 \pm 8.5 -fold higher $(n=4, P=0.01)$ in the total homogenates from dobutamine-treated animals compared to controls (Fig. 5a). In nuclear protein extracts, PY-STAT3 was $6.9 \pm 2.1$-fold higher $(n=4 ; P=0.03)$ in animals treated with dobutamine than in controls (Fig. 5b). In Fig. 5c, the distribution of PY-STAT3 in the different fractions is shown. PY-STAT3 was detectable in all fractions, but the majority of PY-STAT3 was present in the cytosolic fraction. In contrast, the amount of B23 in this fraction was relatively low, which indicates that

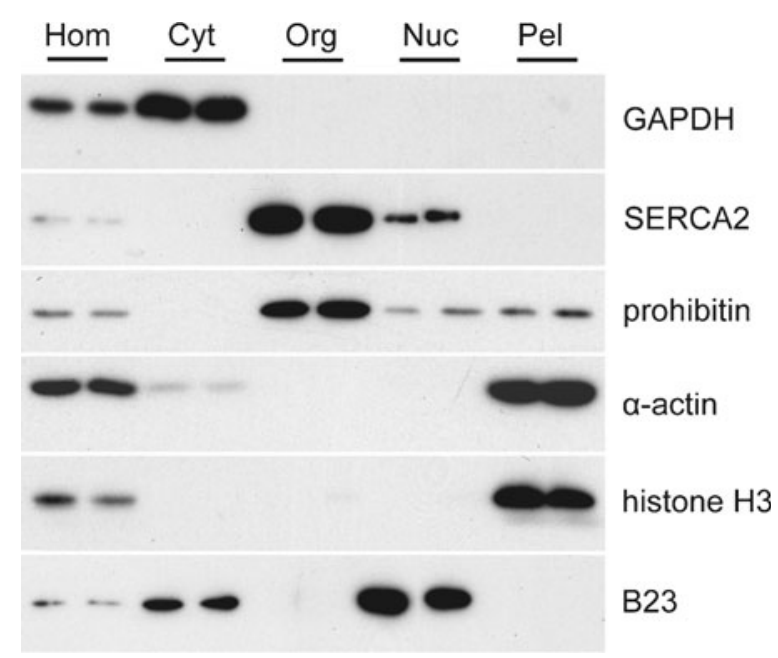

Fig. 4 Subcellular markers in subcellular fractions from frozen LV tissue. Fractionation was performed in parallel on two different frozen LV tissue samples. Of each fraction, $10 \mu \mathrm{g}$ was analyzed by immunoblotting. Hom total homogenate, Cyt cytosolic fraction, $\mathrm{Org}$ organellar fraction, Nuc nuclear protein extract, $\mathrm{Pel}$ final pellet fraction the presence of PY-STAT3 in this fraction is not merely due to leakage from damaged nuclei.

\section{Discussion}

In this report, we describe an optimized nuclear protein extraction protocol for frozen porcine heart tissue. With this relatively simple protocol, we can reproducibly obtain nuclear protein extracts with high enrichment of nuclear proteins, which are essentially devoid of cytosolic, myofilament, and histone proteins. The protein yields of the nuclear fractions are higher in extracts from fresh tissue, but this can be explained by loss of proteins to the cytosolic fraction from nuclei damaged during freeze-thawing as illustrated by increased appearance of B23 in the cytosolic fraction (Fig. 3). We have used B23 as nuclear marker protein. B23 has been reported to shuttle between the cytoplasm and the nucleus in isolated chick or interspecies (mouse-chick) heterokaryon fibroblast cell lines [3]. If shuttling also occurs in porcine cardiomyocytes, the cytosolic appearance of B23 may not only be due to loss of nuclear proteins from
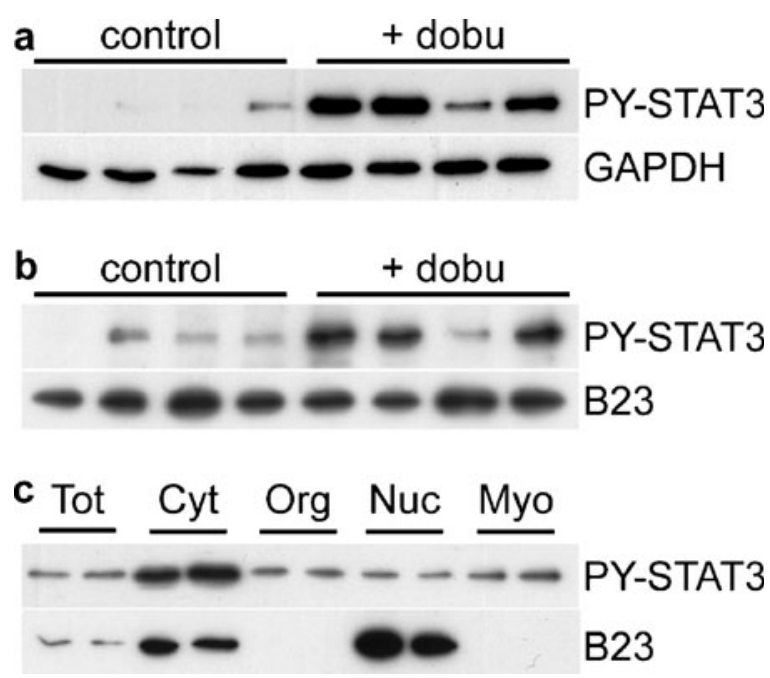

Fig. 5 Effect of dobutamine on PY-STAT3 distribution. PYSTAT3 amount was determined in total homogenates (a) and in nuclear protein extracts (b) from frozen LV tissue of four swine treated without (control) or with dobutamine $(+d o b u)$. GAPDH and B23 were used as loading control, respectively. c Nuclear protein extraction was performed in parallel on two frozen LV samples of swine treated with dobutamine. Of each fraction, $10 \mu \mathrm{g}$ was analyzed by immunoblotting. Hom total homogenate, Cyt cytosolic fraction, Org organellar fraction, Nuc nuclear protein extract, $\mathrm{Pel}$ final pellet fraction 
damaged nuclei, but also to active shuttling, thereby leading to an underestimation of the enrichment of nuclear proteins by our extraction procedure. We show, however, that B23 was almost exclusively localized in the nuclei of porcine LV tissue (Fig. 1). Furthermore, in the nuclear extracts from fresh tissue, where the nuclei are not damaged by freeze-thawing, almost no B23 is seen in the cytosolic fraction (Fig. 3).

When studying transcription factors, it is critical that the cytosolic proteins are removed from the nuclear protein extracts as a number of TFs are present in their inactive form in the cytosol (e.g., NFAT). Using this protocol, the cytosolic marker protein GAPDH was not observed in the nuclear fraction. Since most of the abundant cytoplasmic proteins, i.e., the cytosolic and myofilamental proteins, as well as the most abundant nuclear proteins, i.e., the histones, are removed, the resulting nuclear protein extract appears to be aptly suited for further analysis of $\mathrm{TF}$ abundance and function. We are planning to apply this method to nuclear proteome profiling on remodeled [26] and hibernating [24] myocardium. Since there is minimal contamination of the nuclear protein extract with cytosolic proteins, this method is highly useful for the analysis of nuclear presence of TFs in different conditions.

We tested the applicability of the method by studying the distribution of PY-STAT3 in porcine myocardium after $\beta$-adrenergic stimulation. This stimulation causes phosphorylation of STAT3 and an increase in its transcriptional activity [31]. However, only a small part of the PY-STAT3 pool was found in nuclear protein extracts from dobutamine-treated animals (Fig. 5c), indicating that only a minority of PYSTAT3 translocates to the nucleus. Also, the increase in PY-STAT3 is much higher in the tissue homogenate than in the nuclear protein extracts from dobutaminetreated animals. This demonstrates the necessity to perform nuclear protein extractions when studying the role of PY-STAT3 in the regulation of gene expression.

In a recent study, Murray et al. [22] tested a nuclear extraction protocol on frozen heart tissue and reported nuclear extracts with major contamination from cytosolic GAPDH and mitochondrial ATP synthase subunit $\beta$ marker proteins. One difference with our study is the use of a different tissue homogenization method, which we found to be a crucial step in ensuring proper separation of the fractions. Murray et al. also used a different nuclear extraction kit, using DNAse to cut DNA and release all DNA bound proteins. In contrast, the kit we used employs salt extraction, which likely explains the absence of histone proteins in the nuclear extract.

An inherent limitation of whole tissue homogenization is that it does not allow delineation between nuclear protein extracts from different cell types present in cardiac tissue, including fibroblasts, vascular smooth muscle cells, and endothelial cells in addition to cardiomyocytes. Future studies using laser capture approaches are needed to study nuclear extracts from specific cell types within the heart, but may yield too low levels of transcription factors to be quantified reliably.

In conclusion, the preparation of nuclear protein extracts from frozen tissue allows for the study of nuclear localization of TFs and of TF function in gene regulation, years after tissue has been collected. We used tissue samples that had been stored for 1 week to 10 years and observed no effect of the time of storage on the amount of nuclear protein recovered (data not shown). In addition, using frozen tissue allows for the preparation of nuclear protein extracts from several LV biopsies in parallel, thereby reducing potential day-to-day variability. This method will not only enable the study of nuclear protein profiles of heart tissue from experimental animals present in tissue banks, but may also be applicable to the analysis of human heart biopsies collected in biobanks.

Acknowledgment This work was supported by a grant from the Netherlands Heart Foundation (NHS2005B234).

\section{References}

1. Ahmed N, Rice GE (2005) Strategies for revealing lower abundance proteins in two-dimensional protein maps. $\mathrm{J}$ Chromatogr B Analyt Technol Biomed Life Sci 815:39-50

2. Barthelery M, Salli U, Vrana KE (2008) Enhanced nuclear proteomics. Proteomics 8:1832-1838

3. Borer RA, Lehner CF, Eppenberger HM, Nigg EA (1989) Major nucleolar proteins shuttle between nucleus and cytoplasm. Cell 56:379-390

4. Brown M, McGuinness M, Wright T, Ren X, Wang Y, Boivin GP, Hahn H, Feldman AM, Jones WK (2005) Cardiac-specific blockade of NF-kappaB in cardiac pathophysiology: differences between acute and chronic stimuli in vivo. Am J Physiol Heart Circ Physiol 289:H466-H476

5. Bukowska A, Lendeckel U, Hirte D, Wolke C, Striggow F, Rohnert P, Huth C, Klein HU, Goette A (2006) Activation of the calcineurin signaling pathway induces 
atrial hypertrophy during atrial fibrillation. Cell Mol Life Sci 63:333-342

6. Carlson DL, White DJ, Maass DL, Nguyen RC, Giroir B, Horton JW (2003) I kappa B overexpression in cardiomyocytes prevents NF-kappa B translocation and provides cardioprotection in trauma. Am J Physiol Heart Circ Physiol 284:H804-H814

7. Chong AJ, Shimamoto A, Hampton CR, Takayama H, Spring DJ, Rothnie CL, Yada M, Pohlman TH, Verrier ED (2004) Toll-like receptor 4 mediates ischemia/reperfusion injury of the heart. J Thorac Cardiovasc Surg 128:170-179

8. Cox B, Emili A (2006) Tissue subcellular fractionation and protein extraction for use in mass-spectrometry-based proteomics. Nat Protoc 1:1872-1878

9. Duan S, Yao Z, Hou D, Wu Z, Zhu WG, Wu M (2007) Phosphorylation of Pirh2 by calmodulin-dependent kinase II impairs its ability to ubiquitinate p53. EMBO J 26:30623074

10. Fischer UM, Radhakrishnan RS, Uray KS, Xue H, Cox CS Jr (2009) Myocardial function after gut ischemia/reperfusion: does NF kappaB play a role? J Surg Res 152:264-270

11. Gurusamy N, Malik G, Gorbunov NV, Das DK (2007) Redox activation of Ref-1 potentiates cell survival following myocardial ischemia reperfusion injury. Free Radic Biol Med 43:397-407

12. Huber LA, Pfaller K, Vietor I (2003) Organelle proteomics: implications for subcellular fractionation in proteomics. Circ Res 92:962-968

13. Kislinger T, Gramolini AO, MacLennan DH, Emili A (2005) Multidimensional protein identification technology (MudPIT): technical overview of a profiling method optimized for the comprehensive proteomic investigation of normal and diseased heart tissue. J Am Soc Mass Spectrom 16:1207-1220

14. Laemmli UK (1970) Cleavage of structural proteins during the assembly of the head of bacteriophage T4. Nature 227:680-685

15. Lameris TW, de Zeeuw S, Alberts G, Boomsma F, Duncker DJ, Verdouw PD, Veld AJ, van den Meiracker AH (2000) Time course and mechanism of myocardial catecholamine release during transient ischemia in vivo. Circulation 101:2645-2650

16. Li B, Dedman JR, Kaetzel MA (2006) Nuclear Ca2+/ calmodulin-dependent protein kinase II in the murine heart. Biochim Biophys Acta 1763:1275-1281

17. Lin CC, Lin JL, Lin CS, Tsai MC, Su MJ, Lai LP, Huang SK (2004) Activation of the calcineurin-nuclear factor of activated T-cell signal transduction pathway in atrial fibrillation. Chest 126:1926-1932

18. Liu AM, Lo RK, Wong CS, Morris C, Wise H, Wong YH (2006) Activation of STAT3 by G alpha(s) distinctively requires protein kinase $\mathrm{A}, \mathrm{JNK}$, and phosphatidylinositol 3kinase. J Biol Chem 281:35812-35825

19. Mathews ST, Plaisance EP, Kim T (2009) Imaging systems for westerns: chemiluminescence vs. infrared detection. Methods Mol Biol 536:499-513
20. Melling CW, Thorp DB, Noble EG (2004) Regulation of myocardial heat shock protein 70 gene expression following exercise. J Mol Cell Cardiol 37:847-855

21. Mukhopadhyay S, Shah M, Xu F, Patel K, Tuder RM, Sehgal PB (2008) Cytoplasmic provenance of STAT3 and PY-STAT3 in the endolysosomal compartments in pulmonary arterial endothelial and smooth muscle cells: implications in pulmonary arterial hypertension. Am J Physiol Lung Cell Mol Physiol 294:L449-L468

22. Murray CI, Barrett M, Van Eyk JE (2009) Assessment of ProteoExtract subcellular fractionation kit reveals limited and incomplete enrichment of nuclear subproteome from frozen liver and heart tissue. Proteomics 9:3934-3938

23. Shah M, Patel K, Mukhopadhyay S, Xu F, Guo G, Sehgal PB (2006) Membrane-associated STAT3 and PY-STAT3 in the cytoplasm. J Biol Chem 281:7302-7308

24. Sorop O, Merkus D, de Beer VJ, Houweling B, Pistea A, McFalls EO, Boomsma F, van Beusekom HM, van der Giessen WJ, VanBavel E, Duncker DJ (2008) Functional and structural adaptations of coronary microvessels distal to a chronic coronary artery stenosis. Circ Res 102:795-803

25. Tahepold P, Vaage J, Starkopf J, Valen G (2003) Hyperoxia elicits myocardial protection through a nuclear factor kappaB-dependent mechanism in the rat heart. $\mathrm{J}$ Thorac Cardiovasc Surg 125:650-660

26. van der Velden J, Merkus D, Klarenbeek BR, James AT, Boontje NM, Dekkers DH, Stienen GJ, Lamers JM, Duncker DJ (2004) Alterations in myofilament function contribute to left ventricular dysfunction in pigs early after myocardial infarction. Circ Res 95:e85-e95

27. van Deursen D, Botma GJ, Jansen H, Verhoeven AJ (2008) Down-regulation of hepatic lipase expression by elevation of cAMP in human hepatoma but not adrenocortical cells. Mol Cell Endocrinol 294:37-44

28. von Holt C, Brandt WF, Greyling HJ, Lindsey GG, Retief JD, Rodrigues JD, Schwager S, Sewell BT (1989) Isolation and characterization of histones. Methods Enzymol 170:431-523

29. Wu Y, Tu X, Lin G, Xia H, Huang H, Wan J, Cheng Z, Liu M, Chen G, Zhang H, Fu J, Liu Q, Liu DX (2007) Emodinmediated protection from acute myocardial infarction via inhibition of inflammation and apoptosis in local ischemic myocardium. Life Sci 81:1332-1338

30. Xi L, Taher M, Yin C, Salloum F, Kukreja RC (2004) Cobalt chloride induces delayed cardiac preconditioning in mice through selective activation of HIF-1alpha and AP-1 and iNOS signaling. Am J Physiol Heart Circ Physiol 287: H2369-H2375

31. Zhang H, Feng W, Liao W, Ma X, Han Q, Zhang Y (2008) The gp130/STAT3 signaling pathway mediates betaadrenergic receptor-induced atrial natriuretic factor expression in cardiomyocytes. FEBS J 275:3590-3597

32. Zhao TC, Kukreja RC (2003) Protein kinase C-delta mediates adenosine A3 receptor-induced delayed cardioprotection in mouse. Am J Physiol Heart Circ Physiol 285: H434-H441 